\title{
Sustainable Product Lifecycle Management and Territoriality: New Structure for PLM
}

\author{
Kiyan Vadoudi, Romain Allais, Tatiana Reyes, and Nadege Troussier \\ ICD, HETIC Department, CREIDD, Université de technologie de Troyes, UMR 6281, \\ CNRS, Troyes, France \\ kiyan.vadoudi@utt.fr
}

\begin{abstract}
Product Lifecycle Management (PLM) is a business strategy, which aims to streamline the flow of information about products and related processes throughout the whole product's Lifecycle such that the right information in the right context at the right time can be made available. Recently some studies have been presented on general capabilities of PLM to improve sustainability paradigm, but the subject is still not solved completely. In this paper, first a critical review on the recent advances of Sustainable PLM is presented then a new structure for PLM is proposed based on combination of geographical information systems (GIS) and Lifecycle assessment (LCA), to reach an improvement on sustainable development in territorial scale for achieving a more sustainable paradigm in global scale.
\end{abstract}

Keywords: Product Lifecycle Management, Sustainable Development, Geographical Information Systems, Lifecycle Assessment.

\section{Introduction}

Company, industrial ecosystem and territory are complex systems in interaction that are organized according to their own specific goal. Although these objectives are usually divergent, sustainable development can be a common target for these embedded systems [1]. Recently, there is a shift to manage the environmental and social impacts of products by PLM across the Lifecycle stages, which is called Sustainable Product Lifecycle Management (SPLM) [2-5]. It requires an in-depth understanding of semantics and structure of product Lifecycle information over the whole Lifecycle from technical, social, cultural and geographical implications. Moreover, it is necessary to know which product Lifecycle information is required from where for each operational issue and each Lifecycle phase, and to classify them into several types depending on their characteristics [6].

Among the tools and methodologies available to evaluate the environmental, economic and social performance of materials and consumer products (including their impact on climate change and natural resources), Life Cycle Assessment (LCA) provides a holistic approach that considers the potential impacts from all stages of manufacture, product use and end-of-life [3]. Thus, it could be expectable to have 
different compliance status for a given product cause of different territorial characteristics (proximity to resources, land use, proximity to rivers, etc.) and regulations, which are emphasizing to make link with Geographical Information Systems (GIS).

This research purpose is to link GIS and PLM to have a global and systematic approach to improve company sustainable performance and potential improvement of sustainability at territorial scale. It argues on the necessity to take into account local information concerning where the business takes place. Then, from an information system point of view, it argues the necessity to integrate geographical information in the definition of products and processes, and in the whole PLM. It will lead to an integrated data model including geographic and industrial system data.

\section{Sustainable Development and Territoriality}

Sustainable development issues are manifesting themselves in various forms but an environmental issue is considered as a central point of sustainable development in a spatial setting. It leads to the necessity to specify more precisely the interactions between different resource and industries. Such a more local and regional orientation is mandated not only by the character of the economic and environmental interactions but also by the spatial orientation of policies not only land use. In this regards, some comprehensive impact models based on territory specification have to be developed that encapsulate the complex interacting patterns of regional developments and related consumptions and emissions in relation with social, environmental, economic variables in order to enable industrial decision making. It is noteworthy to mention that the spatial scale of analysis could be handled by Geographical Information Systems (GIS) as a major tool in this field [5]. GIS techniques have been instrumental in developing interactive modes between quantitative modeling and spatial mapping [6] especially when regional development plans have a bearing on the territory, GIS offers a powerful analytical tool for regional/spatial sustainable development [7].

\section{The Geographical Information Systems}

Geography seeks to understand the Earth with all of its human and natural complexities that puts understanding of social and physical processes within the context of places and regions, recognizing the great differences in cultures, political systems, economies, landscapes and environments across the world, and the links between them [8]. Progress within geography as spatial science and integration with technical advances leads to develop a Geographical Information System (GIS), which integrates hardware, software and data for capturing, managing, analyzing and displaying all forms of geographically referenced information. Globally, there are more than 2 million users of GIS and most companies are still unaware of how this technologies influences upon their daily activities [9]. 
A GIS is essentially a tool for decision-making and its powerful analytical and visualization capabilities provide the answers to important questions that must be answered in order to make sound and to inform for decision making. A GIS allows us to develop models, create scenarios and ultimately provide solutions for various environmental and socio-economic problems that exist [9]. Although GIS have been used for several years in the natural resources, forestry, and environmental industries, only recently they have begun to be used for a broader array of business and management functions such as logistics, site and facilities management, marketing, decision making and planning. GIS can help a retail business in locating the best site for its next store and helps marketers in finding new prospects [10].

\section{$4 \quad$ Product Lifecycle}

Product Lifecycle thinking is an attempt to recognize whole stages of a product within the expected Lifecycle. From production engineering perspective, it is related with the development, production and distribution of the product, which can be defined by three main phases: Beginning-of-life (BOL), Middle-of-life (MOL) and End-of-life (EOL) [11].

BOL stage includes the initial design of a product, its development, testing and initial marketing. During design phase by using many tools, techniques and methodologies, designers, planners and engineers initial design of a product is defined and sent to manufacturing phase, which includes production process, plans, production facilities and manufacturing. The BOL stage is crucial to the sustainable product Lifecycle and elements such as the materials chosen and the processes required to create the product all have a significant impact on the product's ultimate environmental footprint. MOL is including external logistic, use and support (in terms of repair and maintenance). In this phase, the product is in the hands of the final product user/consumer and/or some service providers, maintenance and logistic actors [2]. Finally, in EOL, retired products are re-collected and remanufactured for recovery. The product recovery processes consist in collecting, inspection, disassembly, reuse, remanufacturing, recycling, redistribution, and disposal. During $\mathrm{BOL}$, the information flow is quite complete because it is supported by several information systems like CAD/CAM, product data management (PDM), knowledge management (KM). However, the information flow becomes vague or unrecognized after BOL which prevents the feedback of product-related information such as product usage data and disposal conditions, from MOL and EOL back to BOL. Hence, Lifecycle activities of MOL and EOL phases have limited visibility of the product-related information [12] considering the fact that sustainability in product development is tied with closed-loop flow of information.

\section{$5 \quad$ Life Cycle Assessment}

In order to help designers in understanding and translating the environmental constraints into effective actions, Life Cycle Assessment (LCA) methodology has 
been developed to enable the generation of more Eco-efficient design alternatives [3]. Specifically it is a method to assess the environmental impact of a product during its Lifecycle, from the extraction of raw materials to the production and distribution of energy through the use, reuse, recycling and final disposal. LCA is a tool for relative comparison and not for absolute evaluation, thereby it can be used by decision makers to compare all major environmental impacts in the choice of alternative courses of action [13]. The core phase of an LCA analysis is the Lifecycle Inventory compilation that regards the identification of all input and output flows concurring in the product Lifecycle [14]. The procedures of life cycle assessment (LCA) are part of the ISO 14000 environmental management standards: in ISO 14040:2006 and 14044:2006 [15-16].

Regionalization, in the context of LCA, is the recognition that industrial production characteristics and the environmental impact of environmental flows vary among sitegeneric, site-dependent, and site-specific assessments. Site-generic is globally valid, site-dependent operates on the regional scale, and site-specific is only locally applicable [15]. During the LCA modeling each subsystem of the Lifecycle is linked together into a chain of processes, in one end extracting resources and in the other giving various types of emissions or waste. This chain of linked processes is referred to as the technical system. In reality a technical system is under some sort of human control and designed for a certain purpose, to deliver a certain benefit or good, which in the LCA is expressed through the functional unit of the system. The processes are also located somewhere, which implies that they can be geographically referenced. Environmental impact caused by a technical system, or its LCA equivalent, the functional unit, is estimated in terms of the negative change implied by the technical system upon the environmental system, as evaluated by the social system. These systems may also be geographically referenced, which is an important starting point for a consideration of the relations between LCA and localized environmental impacts [14].

\section{Product Lifecycle Management}

Product Lifecycle Management as a business strategy makes it possible to manage the whole Lifecycle of a product. It is ideally an organizationally information and knowledge processing system that integrates information-driven approach comprised of people, processes/practices and technology to all aspects of a product's life and provides a central system to centralize product data, standardize business processes and streamline communication of information across distributed product development teams to shorten development cycles, improve quality and speed time-to-market [21]. To be able to plan the implementation of PLM effectively into an organization, it needs to be understood also the strategic aspect of PLM far more than just an IT system, to develop the company as a whole to integrate all organizational aspects and levels. In this regard, Anneli Silventoinen [21] presented a holistic PLM model that includes five elements of strategies defining main approaches; operational processes of the value chain; structures of product, knowledge and organization; people and culture, and information technology means. 
Since Information and Communication Technology (ICT) plays an important role in PLM, the ICT architecture of a company and its dynamic adaptation to new technological developments is necessary for realizing the full PLM potential. ICT is used in running the PLM processes and in up-dating of data and information structures, mainly for creation, acquisition, storing, sharing and application of documented knowledge, but also for collaboration. In this regards different IT tools, platform and systems that are spread through complete Lifecycle of product are dividing in two categories of "ICT tools and systems" and "ICT interoperability and architectures"'[2].

Despite progresses in computer-aided design (CAD), computer-aided manufacturing (CAM), computer-aided process planning (CAPP), product data management (PDM), enterprise resource planning (ERP), Lifecycle Assessment (LCA), which help to make decision in the design process, there are still shortcomings in this stage because they were usually separated from a manufacturing company's mainstream operations and they cannot adequately address the need for collaborative capabilities throughout the whole product Lifecycle and they usually focus on special activities in an enterprise, which are not adequately designed to meet new business requirements. Especially, Lifecycle Assessments (LCA) can feed PLM to improve global performance in a sustainable framework, but to have a represent-ability of the model, it may be necessary to have the input data as specific as possible including geographic specificities [2].

\section{$7 \quad$ Sustainable Product Lifecycle Management}

During recent years there is a shift to talk about Sustainable Product Lifecycle Management (SPLM) as an opportunity applying scientific knowledge to design and implementing products and processes. N. Duque Ciceri [18] introduced PLM as a platform to provide sustainability by continuous sharing of information among the different product Lifecycle phases. He believes, there are two main challenges for doing PLM as an effective tool for Sustainable Manufacturing; "closing product Lifecycle information loops" and "defining a reference model for the PLM approach to sustainability and Sustainable Manufacturing in particular". Kary Framling [19] by emphasizing more on environmental dimension based on closed-loop PLM, introduced intelligent products as an approach to reduce $\mathrm{CO} 2$ emissions, energy usage and environmental damage through the communication between products and other systems. In that paper closed loop Lifecycle management is defined as a basis approach for SPLM to improve environmental sustainability during all phases of the Lifecycle it uses Life Cycle Assessment (LCA) as a methodological framework for estimating and assessing the environmental impacts and "energy informatics" for analyzing the flow of energy between components of the whole system. Martin Eigner [17] proposed a solution for monitoring the sustainability of product development processes based on Integrated Sustainability Triangle. The Integrated Sustainability Triangle, originally introduced as a promising new possibility of quantification and monitoring the Sustainable Development of a national economy, is also an appropriate instrument for the systemization and evaluation of the performance of a company regarding sustainability management. In next section a new structure for PLM is proposed, which tries to be better support of sustainability strategy. 


\section{Improved PLM structure}

This research methodology is based on a mere literature study, evaluating the potential of using GIS in PLM structure by use of LCA as an interrelation tool to improve sustainability in territorial scale, which can be seen as a pre-study to demonstrate and illustrate the impact of taking account of geographic information in PLM to design sustainable systems. Review of related works presented above about SPLM indicate that current PLM solutions for supporting sustainability are often not adequate for the management of product design information beyond BOL, MOL and EOL stages. One of the major problems about implementation and evaluation of the sustainable development is the lack of specificity in concrete circumstances. It means sustainable development, which is implemented in a region, is not necessarily sustainable elsewhere and a large number of small-scale and marginal changes at the local or regional level have clear global dimensions. Thus, accessibility to geographical information can play critical role on quantification and monitoring sustainability in specific area and then running product development process based on results of sustainability assessment. Therefore, it is essentially required to identify and develop a technology for current PLM structure as beginning in company scale to enable product development process based on the result of sustainability in regional scale, in order to solve the issue of sustainability in global scale.

The fact that businesses can start to use GIS within PLM is not surprising, particularly given the fact that much of the data that organizations typically use include significant spatial components (estimates range between 50\% and 85\%) [9]. Researchers and professionals have seen the potential of using GIS in the planning for a sustainable development; by mapping the same factor in different time spans and spatial areas an overview of changes is created and hence make it easier and more correct to predict future changes and make well founded planning decisions in urban areas [22]. By implementing GIS, "Territory" in macro level adds to the structure of

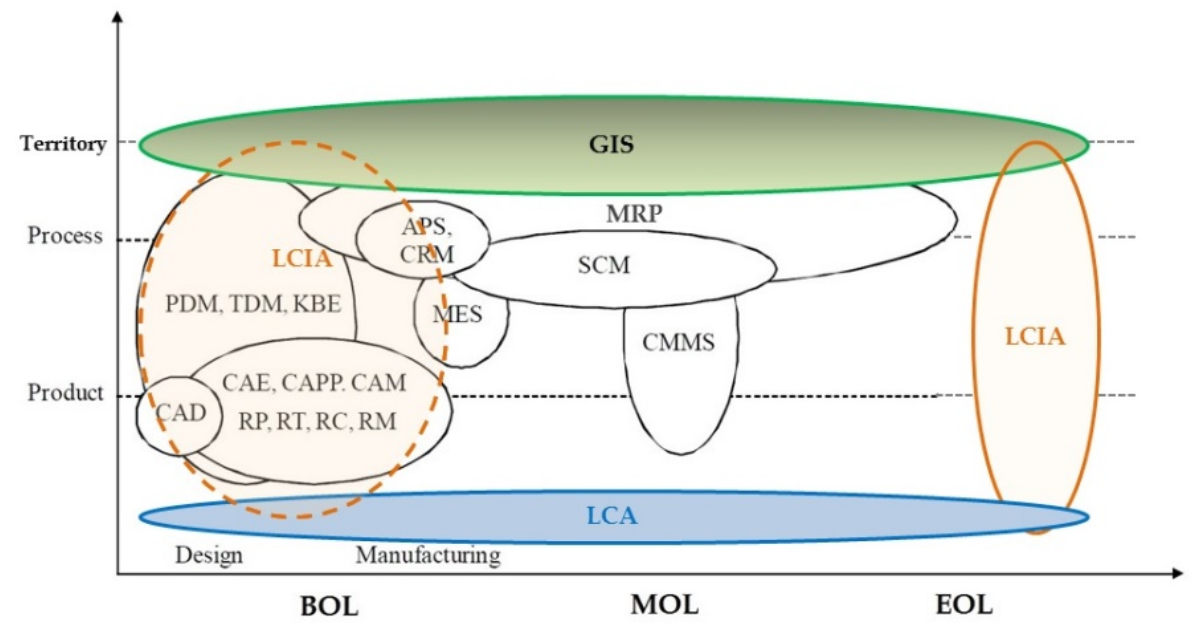

Fig. 1. Improved ICT structure in product lifecycle 
PLM and makes it able to provide a proper catalog of the surroundings including environmental impact on natural ecosystems, transportation, community demographics, public safety, utilities, services and accessibility (Fig.1).

The nature of each geographical aspect is presented in the structure of $L C A$ as the three subsections of technical, environmental and social systems, which make link between "production" and "process" scales with "territory" through the results of LCIA. A technical system generally includes processes which might be geographically referenced, and which are connected via different types of transport systems, such as goods (road, sea, air) or energy ware (pipelines, electricity grid) distribution systems. The geographic location and extension of such a technical system gives relevant information for the modeling and assessment of its environmental impact [23]. By giving the geographical location of the different parts of the technical system, it will be possible to model the dispersion of various agents, so that the varying sensitivity of ecosystems, regions, etc., can be taken into account where this is relevant. A geographically large technical system and the environmental impacts of such a system may cross national, regional and even continental boundaries, and therefore also affects different cultures or groups of people, holding different attitudes towards changes in the environment.

The amassing of these tangible elements of a community goes a long way toward informing the design process, and should always be considered to look at efficiency and sustainability. For instance, by using this new structure it would be possible to develop companies' energy consumption, water usage, $\mathrm{CO} 2$ emissions and wastes generation to build scenarios, assess them and make decision. When it comes to model, it will able companies to locate points of water usage, transportation of water from its source to its consumption point, distance of water transportation, climate change, air pollutions, soil-map, location of landfill, customers distribution and other information related with Lifecycle that can be useful in $B O L$ to design product, process and plant (Fig.2). It will enable to enhance circular economy and industrial ecology.

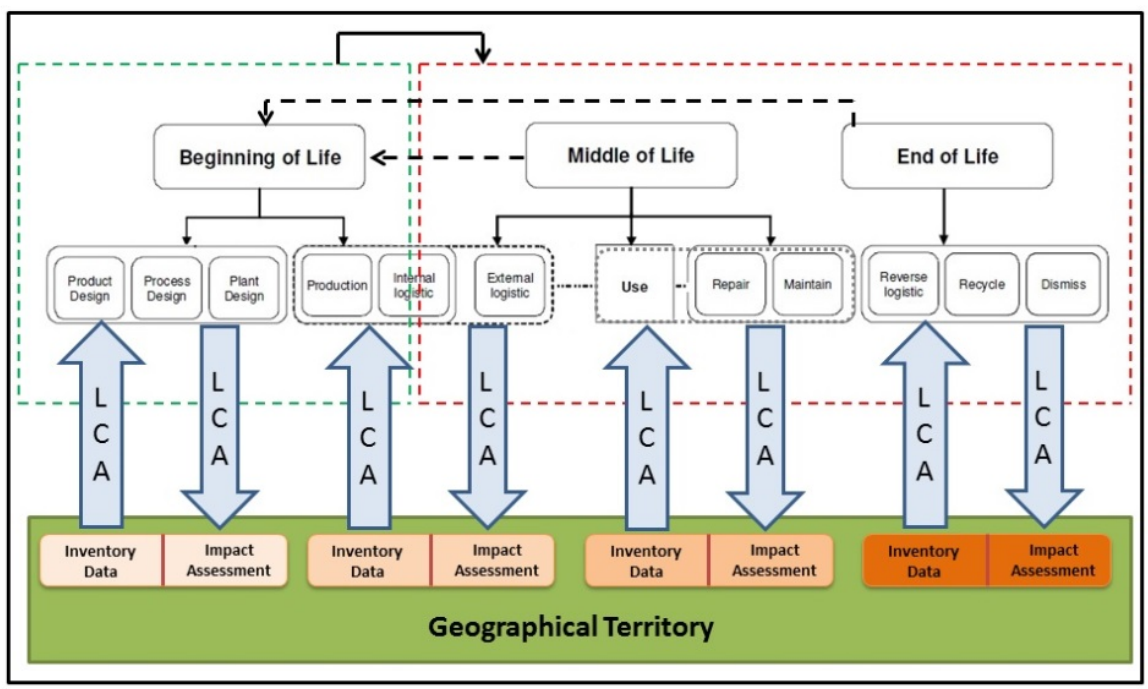

Fig. 2. Interaction between Product lifecycle, GIS, LCA and LCIA 
By using sustainability monitoring, it will be possible to analysis the result of this new PLM structure. Several solutions for the monitoring of sustainability of product development processes have been developed but the one that is much more related with PLM, is proposed by Martin Eigner based on integrated sustainability triangle [17] by a weighted aggregation of the single indicators to define the asserted contribution of a respective field. Each field includes several indicators that may contribute to a different extent to the outcome for that field but together all used indicators illustrate $100 \%$ of the subject field. The result can present as an Integrated Sustainability Triangle to monitor the sustainability performance and supports communication via graphic elements. There is a shortcoming with this representation as every dimension of this triangle is dependant to another one. Moreover the single score evaluation of the three dimensions of sustainability is a "black box" and data needed locally are not directly reachable.

To overcome this issue of reuse of the information, the French national agency (ANR) founded Convergence project propose a multi-level management system [24]. The three-organization level of a company (i.e. top management, middle management and operations) and their respective domain of action (i.e. strategy, tactics and operation) are connected by a set of indicators dedicated to their daily activities. A dashboard extended to intangibles allows the evaluation and use of local and global indicators to manage the positive and negative externalities of a company (I.E. sustainable performance). A future development is to systematize data collection by connecting to PLM. When available, Convergence enables global performance management for companies.

Thus, it is essentially required to identify and develop a technology for current PLM structure to enable product development process based on the result of sustainability in regional scale, in order to solve the issue of sustainability in global scale.

\section{Conclusions}

There is no doubt that there is a lot of complexity in the sustainability concept. A possible reduction of this complexity is a proper management, planning and design actions. Therefore, it can be concluded that a holistic management of resources is presented in both consumption and production activities. The goal is to reach from a micro scale of design to macro scale to expand boundary beyond the factory wall to optimize the resources utilization. In macro level businesses measure the sustainability level of same industry or territory to make decision for their design stages and this means they don't think about themselves alone, but there is a higher level entity who is managing the sustainability as a whole. Vertical integration of industries, cross industry mix and community involvement could be subjects for further studies, in order to bring them into PLM structure by using geographical information as driven cross industries.

In summary, the paper shows that GIS should be included in PLM framework to localize environmental, economic and social, related to specific locations when designing green manufacturing systems. 
It will lead to the definition of an integrated framework (integration of geographic and industrial data) in order to support decision making, especially in BOL. It will enable both:

- Industrial ecology principles to be applied in BOL reasoning;

- Environmental impact assessment with respect to the local context.

\section{References}

1. Capron, M., Quairel, F.: Évaluer les stratégies de développement durable des entreprises: l'utopie mobilisatrice de la performance. l'organisation Responsable (2006)

2. Terzi, S., Bouras, A., Dutta, D., Garetti, M.: Product lifecycle management-from its history to its new role. Int. J. Prod. Lifecycle Management 4, 360-389 (2010)

3. Hart, A., Clift, R., Riddlestone, S., Buntin, J.: Use of Life Cycle Assessment to Develop Industrial Ecologies, A Case Study 83(4), 359-363 (2005)

4. Glavič, P., Lukman, R.: Review of sustainability terms and their definitions. J. Clean. Prod. 15(18), 1875-1885 (2007)

5. Burrough, P., McDonnell, R.: Principles of geographical information systems (1998)

6. Maria Giaoutzi, P.N.: Decision support models for regional sustainable development, p. 304. Avebury, Brookfield, Vt, Ashgate Pub. Co., ldershot, Hants, England (1993)

7. Nijkamp, P., Ouwersloot, H.: A decision support system for regional sustainable development: the flag model. Tinbergen Institute Discussion Paper (1997)

8. Johnston, R.: Geography and GIS. Geogr. Inf. Syst. Princ, pp. 39-48 (1999)

9. Azaz, L.: The use of Geographic Information Systems (GIS) in Business. In: Int. Conf. Humanit., pp. 299-303 (2011)

10. Pick, J.: Geographic information systems in business. IGI Global (2004)

11. Kiritsis, D., Bufardi, A., Xirouchakis, P.: Research issues on product lifecycle management and information tracking using smart embedded systems. Adv. Eng. Informatics 17(3-4), 189-202 (2003)

12. Niemann, S., Tichkiewitch, Westkämper, E.: Design of sustainable product life cycles. Springer (2008)

13. Finnveden, G., Hauschild, M.Z., Ekvall, T., Guinée, J., Heijungs, R., Hellweg, S., Koehler, A., Pennington, D., Suh, S.: Recent developments in Life Cycle Assessment. J. Environ. Manage. 91(1), 1-21 (2009)

14. Mutel, C.L., Pfister, S., Hellweg, S.: GIS-based regionalized life cycle assessment: how big is small enough? Methodology and case study of electricity generation. Environ. Sci. Technol. 46(2), 1096-1103 (2012)

15. ISO, 2006a. ISO 14040 International Standard. In: Environmental Management - Life Cycle Assessment - Principles and Framework. International Organisation for Standardization, Geneva, Switzerland

16. ISO, 2006a. ISO 14044 International Standard. In: Environmental Management - Life Cycle Assessment - Principles and Framework. International Organisation for Standardization, Geneva, Switzerland (2006a)

17. Eigner, M., Von Hauff, M., Schäfer, P.: Sustainable Product Lifecycle Management: A Lifecycle based Conception of Monitoring a Sustainable Product Development. Glocalized Solut. Sustain, pp. 2-7 (2011)

18. Ciceri, N.D.: Product lifecycle management approach for sustainability. In: Proc. 19th, pp. 30-31 (March 2009) 
19. Främling, K., Holmström, J., Loukkola, J., Nyman, J., Kaustell, A.: Sustainable PLM through Intelligent Products. Eng. Appl. Artif. Intell. 26, 789-799 (2013)

20. Dyllick, T., Hockerts, K.: Beyond the business case for corporate sustainability. Bus. Strateg. Environ. 11(2), 130-141 (2002)

21. Silventoinen, A., Pels, H., Kärkkäinen, H., Lampela, H.: Towards future PLM maturity assessment dimensions (2011)

22. Walsund, E.: Geographical Information Systems as a Tool in Sustainable Urban Development, Malmö University (2012)

23. Vadoudi, K., Troussier, N., Zhu, T.W.: Sustainable Manufacturing through PLM, GIS and LCA interaction. In: International Conference on Engineering, Technology and Innovation, Bergamo, Italy (2014)

24. Zhang, F., Rio, M., Allais, R., Zwolinski, P., Carrillo, T.R., Roucoules, L., Buclet, N.: Toward a systemic navigation framework to integrate sustainable development into the company. Journal of Cleaner Production 54, 199-214 (2013) 